\title{
CONSERVATION DU LAIT PAR DES MÉTHODES AUTRES QUE LES MÉTHODES CLASSIQUES
}

\author{
par \\ Georges RAY
}

Professeur honoraire d'Ecole nationale d'Agriculture,

Ex-chef du Service technique à l'Institut international d'Agriculture de Rome

L'industrie laitière est entièrement dominée par la nécessité d'assurer, à tout instant, une conservation satisfaisante des produits à traiter. On s'est ingénié en conséquence, à tirer le meilleur parti possible des méthodes classiques, permettant de prolonger plus ou moins la période d'utilisation du lait.

- Ces méthodes sont maintenant bien au point, et leur mise en œuvre dépend avant tout du but poursuivi, notamment de la durée de conservation qu'il s'agit d'atteindre. On sait que les procédés de conservation applicables au lait, dans la pratique, se ramènent à cinq groupes :

1. Conservation par la chaleur.

2. Conservation par le froid.

3. Conservation par dessication.

4. Conservation par occlusion de l'air.

5. Conservation par les antiseptiques.

Cette liste n'est pas limitative, et l'on caresse volontiers l'espoir de perfectionner les méthodes classiques, de combiner heureusement certaines d'entre elles, ou même de les abandonner complètement, en leur substituant des méthodes entièrement nouvelles.

C'est ainsi qu'on s'oriente depuis peu, vers l'utilisation de méthodes nouvelles qui font naître de grands espoirs dans les milieux laitiers. Certains voient déjà la pasteurisation détrônée, ce qui est aller un peu trop vite en besogne.

Tout en accordant aux méthodes nouvelles l'attention qu'elles méritent, il sera prudent de se faire une opinion, et pour cela d'attendre que les recherches actuellement en cours deviennent applicables, à l'échelle industrielle. On peut prévoir que la solution des problèmes posés n'est certainement pas pour demain.

Il nous a paru qu'une étude d'ensemble rendrait quelques services. Les traitements dont nous allons parler ne font pas appel à des moyens thermiques. Il est done absolument incorreet de les assimiler, comme le font beaucoup de publicistes, en France comme aux Etats-Unis et en Grande-Bretagne, à la pasteurisation.

Nous nous proposons d'examiner successivement : la conservation du lait par l'action oligodynamique de certains métaux, la 
destruction des spores bactériennes par action mécanique, la conservation du lait par l'action des rayons ultra-violets, la conservation du lait par les ultra-sons, la stérilisation électronique du lait.

\section{Conservation du lait par l'action oligodynamique de certains métaux}

Les métaux lourds et les métaux précieux possèdent un pouvoir bactéricide facile à mettre en évidence, en plaçant une monnaie de cuivre ou d'argent sur la gélose ensemencée d'une boîte de Pétri. Le développement des microbes est empêché dans une zone annulaire d'un demi centimètre autour du métal.

Cette oligodynamie négative a servi de base à un procédé de stérilisation de l'eau par l'argent, qui donne des résultats très discutés pour les produits alimentaires liquides.

Sur la possibilité d'appliquer l'action oligodynamique de l'argent à l'industrie des conserves alimentaires, V. D. SLavine [1] a donné des précisions qui ne laissent aucun espoir aux partisans de la méthode. Il déclare en effet que le prétendu effet oligodynamique de l'argent (dans le sens de l'action bactéricide de doses infinitésimales du métal) n'existe pas en réalité, même dans le cas de l'eau. Les doses d'argent qui sont bactéricides dans le cas où l'on étudie l'eau seule, stimulent au contraire la croissance des bactéries au sein d'une substance organique (produit alimentaire). L'argent ne convient donc ni à la stérilisation d'aliments organiques, ni à la stérilisation des eaux utilisées pour le lavage dans les industries de l'alimentation.

\section{Destruction des spores bactériennes par action mécanique}

Cette méthode originale de destruction a été découverte par H. R. Curran et F. R. Evans [2]. En agitant des suspensions de spores bactériennes avec de petites perles de verre (570 secousses par minute), on constate, après six heures d'agitation, que le nombre des spores est passé de un million à trois unités par centimètre cube. Avec des fragments de carborandum à angles vifs, l'effet est moins bon. En expérimentant sur des colibactéries en suspension dans l'eau, la stérilisation complète fut obtenue après cinq heures d'agitation. Dans les mêmes conditions, mais avec du bouillon, 0,01\% des bactéries ont survécu. L'effet de destruction sur les formes végétatives est encore plus complet que sur les spores, ce qui est à retenir.

Bien qu'une semblable méthode de stérilisation ne soit utilisable que dans des cas particuliers et à petite échelle, elle peut cependant trouver des applications intéressantes, notamment l'extraction des endoenzymes (comme l'invertase de la levure). A notre avis, ce 
champ d'application serait susceptible-d'intéresser l'industrie fromagère, la technique de fragmentation bactérienne pouvant parfaitement être appliquée aux bactéries de la maturation, dans le but d'obtenir une maturation accélérée des fromages.

Signalons enfin que la méthode est déjà utilisée, aux EtatsUnis, pour stériliser à froid certaines préparations médicales.

\section{Conservation du lait par l'action des rayons ultra-violets}

Jusqu'à ce jour, l'application la plus intéressante des rayons ultra-violets, en laiterie, se rapporte à l'accroissement de la teneur en vitamine D (irradiation vitaminisante), sujet que Le Lait a bien souvent traité. Or les rayons ultra-violets exercent également une action bactéricide certaine, que divers expérimentateurs cherchent déjà à utiliser, en remplacement de la pasteurisation classique.

Comme l'explique Demeter [3], l'effet bactéricide des rayons ultra-violets se manifeste surtout pour les longueurs d'onde comprises entre 2650 et $2537 \mathrm{~A}^{\circ}$. Les formes végétatives sont plus sensibles que les spores. Les rayons ultra-violets empêchent d'ailleurs la sporulation. Le temps d'élimination varie de plusieurs minutes à des fractions de seconde, selon l'espèce bactérienne et la loi de Bunsen-Roscoe (constance du produit : temps $\times$ intensité) est valable.

Selon LembKE et ses collaborateurs, l'action stérilisante est maximum à la température la plus favorable à la croissance. Mais on retiendra que, tout comme on le constate pour les poisons chimiques, une intensité d'éclairement inférieure à celle qui serait mortelle exerce une action favorisante sur la croissance, probablement par formation d'activeurs.

L'action bactéricide des radiations ultra-violettes se constate aisément, si le lait s'écoule doucement en couche mince. Elle est moins marquée (à égalité d'épaisseur) sur le lait en repos, comme le constatent D. C. Supplee, G. E. Flanigan et O. G. Jensen [4]. Les radiations ultra-violettes courtes $\left(2.200\right.$ à $\left.2.300 \mathrm{~A}^{\circ}\right)$ donnent des résultats bactéricides irréguliers.

En combinant l'action des rayons ultra-violets et celle de la chaleur, pendant un temps inférieur à une seconde, on obtient un effet léthal renforcé.

En quoi consiste l'action exercée par les rayons ultra-violets sur 1a cellule bactérienne ? Il semble qu'il y ait détérioration du protoplasma cellulaire. Déjà, en 1910, CernovodeANu et HeNRI avaient montré qu'après exposition à la lumière ultra-violette, les bactéries ne prenaient plus la coloration. On a constaté aussi la bactériolyse des cellules, ce qui n'exclut pas l'action d'autres facteurs, tels que l'inactivation des enzymes (celle de la catalase est prouvée). 


\section{Application industrielle des propriétés bactéricides des rayons ultra-violets}

Peut-on passer des observations de Iaboratoire à la stérilisation industrielle du lait ? On se heurte, dans la pratique, à deux obstacles. Tout d'abord, certaines lampes de quartz ont le défaut de produire, en même temps que les rayons germicides, des rayons courts générateurs d'ozone, à partir de l'oxygène atmosphérique et de l'oxygène dissous dans le lait. Pour éviter cette réaction, le Dr SchoLL refroidit le lait à $4^{\circ} \mathrm{C}$. et l'emmagasine dans un tank, où l'on chasse l'oxygène par injection d'anhydride carbonique sous pression. Le lait passe alors dans un manchon métallique sous forme d'une pellicule d'un millimètre d'épaisseur, exposée à l'action des rayons ultraviolets. Le lait sortant est recueilli dans un réservoir et va ensuite à l'embouteillage.

Une firme de Hambourg avait, en 1940, monté une installation traitant de 1.000 à 5.000 litres de lait à l'heure, avec exposition de 40 secondes à des rayons de longueur d'onde $2.537 \mathrm{~A}^{\circ}$. On obtenait une stérilisation à $98 \%$, sans apparition de goût ni d'odeur. Un autre dispositif consistait à faire agir les rayons ultra-violets sur un brouillard de lait obtenu par pulvérisation du liquide sous pression de gaz carbonique.

Les techniciens anglais estiment que ce qu'ils appellent bien à tort " la pasteurisation à froid du lait au moyen des rayons ultraviolets " [5] constitue l'une des découvertes les plus importantes réalisées par l'industrie laitière allemande, au cours des dernières années. La technique a été mise au point par les ingénieurs de la firme Siemens. A la fin de la guerre, deux machines fonctionnaient dans une usine de Lubeck spécialisée dans la fabrication du lait en poudre. La plus petite des machines possédait un serpentin de quartz (d'un centimètre de diamètre et cent dix mètres de longueur) dans lequel le lait était soumis à l'action de quatre lampes en tube à vapeur de mercure. Cette machine a servi à l'irradiation vitaminisante.

L'autre machine, de type industriel, permettait en outre d'éliminer la flore bactérienne du lait. Le lait circulait dans une cinquantaine de tubes de quartz, de deux mètres de long, reliés par des tubes en $\mathrm{U}$ en résine de polyvinyle (diamètre intérieur des tubes : un centimètre ; épaisseur de la paroi : un millimètre et demi à deux millimètres). Le lait circulant dans ces tubes était soumis à l'action des rayons ultra-violets émis par des lampes tubulaires à vapeur de mercure. La plus grande difficulté dans une installation de ce genre, est de réaliser une circulation régulière du lait, assurant l'égalité de traitement de toutes les parties du liquide. On a constaté une destruction satisfaisante des bactéries, spores comprises. 
Signalons que nous avons pu voir, en août 1949, une installation en cours de montage à l'Institut des recherches laitières de Kiel. L'appareillage fonctionnera sous la direction du $\mathrm{P}^{\mathbf{r}}$ MонR.

Le second écueil à éviter se rapporte à la pénétration insuffisante des rayons germicides dans le lait à traiter. On constate en effet que les rayons ultra-violets ne pénètrent pas profondément dans le lait. De petites quantités de colloïdes arrêtent l'action des rayons et protègent les bactéries. Selon les conditions d'application, on aboutit à des résultats très variables : parfois nuls, parfois réduction du pourcentage des bactéries des laits crus de mélange supérieure à $90 \%$ et atteignant même 95 à $98 \%$ (SuPPLEe, FlanigaN et Jensen) ou $99,8 \%$ (NrCholson).

Pratiquement, il faut convenir que la réalité est moins brillante. Si, déclare Demeter, on voit dans les cas les plus favorables disparaître $85 \%$ de la flore bactérienne, on est loin de pouvoir assimiler l'action des rayons ultra-violets à une pasteurisation, encore moins à une stérilisation. C'est cette assimilation que n'hésite pas à faire E. Nicholson [6], dans une étude intitulée : "Pasteurisation par l'ultra-violet ". Le terme est absolument impropre, la pasteurisation étant, répétons-le, un traitement thermique.

La mise au point d'un appareillage satisfaisant pour le traitement industriel du lait a été longue, car il faut opérer sur un film liquide très mince, si l'on veut obtenir une réduction importante $d u$ contenu bactérien du lait. On est ainsi conduit à construire des appareils de dimensions considérables. Avant la guerre, NichoLson arrivait à tuer $99,8 \%$ des bactéries du lait, mais avec un rendement par lampe de trois gallons à l'heure (à peine 15 litres). Les premiers essais ont été faits avec une lampe montée au centre d'un appareil cylindrique de métal.

A la fin de 1945, les travaux ont été repris, en utilisant un appareil assurant la formation d'un film liquide, par laminage du lait entre des cylindres horizontaux, montés par paires, dans une sorte de grande

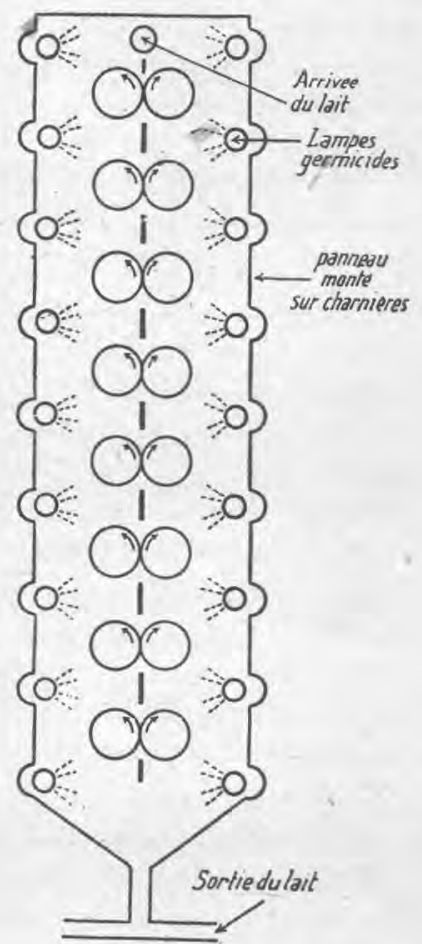

Fig1

schema de l'appareil NICHOLSON. 
boîte à section rectangulaire (fig. 1). Le lait arrive entre les deux premiers cylindres et se trouve entraîné par la rotation. Le film, très mince qui se forme (1/64 d'inch, soit $0 \mathrm{~mm} .4)$ est détaché par une raclette, et le liquide arrive entre les deux eylindres suivants. La vitesse de rotation des cylindres est juste suffisante pour provoquer l'entraînement du liquide.

Les lampes germicides sont placées sur les panneaux latéraux, montés sur charnières, pour permettre l'ouverture et le nettoyage. Ce nettoyage se fait avec plusieurs seaux d'eau, sans démonter les lampes. Les organes en contact avec le lait sont en acier inoxydable ; les panneaux sont en aluminium.

Le lait traité sort à la partie inférieure de l'appareil et va à l'embouteillage. Nicноцsоn estime que son appareil peut être utilisé à la ferme productrice de lait et qu'il permet de supprimer l'opération coûteuse de la pasteurisation. Cette vue optimiste nous semble prématurée, et nous partageons l'avis de Demeter. Dans l'état actuel des choses, écrit-il, il ne saurait être question de remplacer la pasteurisation du lait de consommation par le traitement aux rayons ultra-violets. Même au cas où la réduction du nombre des germes serait aussi importante que dans la pasteurisation, il manquerait la certitude d'une destruction totale des germes pathogènes. Et c'est cela qui compte avant tout.

\section{Renforcement de l'action des rayons ultra-violets par celle des rayons infra-rouges}

On a songé à renforcer l'effet bactéricide des rayons ultra-violets par le rayonnement calorifique de l'infra-rouge. Dans le procédé de la « Fromray Parent Co », l'irradiation est combinée au chauffage, grâce à l'immersion des lampes au sein du lait à traiter. Si la couche de lait est mince, un temps d'exposition de quarante secondes ferait disparaître $98 \%$ de la flore bactérienne (DENNINGTON).

\section{Conservation du lait par les ultra-sons}

Les ultra-sons représentent des vibrations de fréquence si élevée qu'elles ne peuvent être perçues par l'oreille. Les ondes ultra-soniques, généralement produites par des quartz piézo-électriques, doivent être transmises au travers $d u$ corps à traiter (gaz, liquide ou solide), mais non à travers le vide. On les utilise de plus en plus en physiologie et en bactériologie, en partant de cette observation, faite en 1927 par WooD et Loomis, que les ultra-sons exercent un effet destructeur sur toute cellule vivante. L'année suivante, HARVEY et Loomis constataient le même effet sur les bactéries.

La destruction des bactéries en suspension dans un liquide dépend de la fréquence des vibrations et du temps d'application. 
Elle est favorisée par la limpidité du liquide (BECKWITH et WEAVER, 1936) et par les gaz dissous. Mais la destruetion des bactéries par les ultra-sons est toujours incomplète et elle est lente (plusieurs minutes). De plus, certaines bactéries sont résistantes (Microbacterium, Sarcina). On doit done s'attendre à rencontrer de grandes difficultés, dès qu'on tente de réaliser pratiquement la stérilisation par les ultra-sons d'un liquide d'origine organique comme le lait.

Le mécanisme de la destruction des bactéries par les ultra-sons est encore ignoré. On a invoqué notamment :

a) Une action mécanique, attribuable au rythme élevé des vibrations et que certains comparent à l'effet alternatif du gel et du dégel ;

b) Des transformations chimiques du milieu, les unes provenant de la destruction des diastases essentielles, les autres de la formation d'un agent oxydant qui agirait surtout sur les graisses;

c) Des modifications physiques telles que la dénaturation des protéines à la surface des cellules.

Essais de conservation du lait par les ultra-sons. Les premières tentatives de stérilisation du lait par les ultra-sons ont été faites par L. A. Chambers et N. Gaines [7]. Le lait s'écoulait à la surface d'un tube de nickel, les vibrations mécaniques étant transmises par un oscillateur à soupape. Sur du lait eru commercial, on obtint la destruction de $80 \%$ des bactéries, avcc des températures n'excédant pas $10^{\circ} \mathrm{C}$.

BECKWITH et WEAVER sont arrivés à des résultats très variables, la destruction des bactéries dans le lait pouvant aller de $20 \%$ après quinze minutes à $99 \%$ après cinq minutes, les variations en efficience étant probablement dues à divers composants du lait, la caséine notamment.

P. K. StumpF, D. E. Green et F. W. Smith [8] ont constaté que la désintégration des bactéries s'affaiblit à mesure qu'augmente la concentration des cellules; ils ont noté aussi l'action retardatrice des protéines.

Enfin, il est intéressant de signaler que l'emploi des ultra-sons a permis à K. Demeter [9] d'obtenir une diminution sensible de la teneur bactérienne du lait, sans toutefois parvenir à la stérilité complète. Il semble que ce soit l'albumine du lait qui réduit la puissance bactéricide des ultra-sons. L'effet stérilisateur constaté est total pour les Coli, les bacilles typhique et dysentérique, partiel pour les coques, streptocoques et bactéries du foin, mais nul pour les bactéries de l'anthrax.

$\mathrm{Au}$ total, on est encore loin de pouvoir garantir la stérilisation du lait traité. On remarquera en outre que si les vitamines B I 
et $\mathrm{C}$ ne sont pas touchées, le traitement du lait par les ultra-sons provoque diverses transformations de l'état physico-chimique du lait, notamment :

1. Une homogénéisation qu'on peut considérer comme heureuse.

2. Une modification de saveur, due vraisemblablement à un commencement d'hydrolyse des graisses.

3. Une modification de la valeur nutritive, attribuable aux changements provoqués dans la nature des constituants du lait (par exemple l'oxydation des graisses, la dénaturation des protéines).

4. Une action de la chaleur : en effet, l'énergie des ondes ultrasoniques se convertit en chaleur. En conséquence, il faut, ou bien refroidir le lait traité ou accepter les effets de la chaleur.

\section{Stérilisation électronique du lait}

Le bombardement électronique de diverses matières alimentaires aboutit à une stérilisation, obtenue sans modification sensible du milieu. Les impulsions électroniques de faible durée et de grande intensité sont obtenues par l'emploi d'un générateur étudié en 1934 par E.-MARX et R. Brasch, puis aux Etats-Unis par W. HUBer et connu sous le nom de "capacitron".

Pour des produits alimentaires en boîtes (dans lesquelles on n'a pas fait le vide), la disparition complète de la flore microbienne est obtenue avec deux à six impulsions électroniques agissant à travers la paroi des récipients. Pour un liquide en repos, la profondeur de pénétration dépend du voltage, de la nature du liquide, et de la distance du générateur de flux électronique.

L'application de cette méthode nouvelle au cas du lait et des produits laitiers soulève des problèmes à peine effleurés jusqu'à ce jour. On peut redouter des modifications de saveur, notables pour le beurre et le fromage, mais non encore constatées pour le lait.

On pense qu'il y aurait intérêt à combiner la stérilisation électronique avec la conservation par le froid. En tous cas, une voie nouvelle s'ouvre devant les chercheurs, dont l'exploration est à peine amorcée.

\section{Avenir des méthodes de conservation du lait par l'utilisation de diverses radiations}

- La simplicité et la rapidité d'emploi des radiations ont fait naître récemment de grands espoirs. Certains entrevoient même une révolution complète dans la technique de la stérilisation du lait et l'abandon de la pasteurisation. C'est aller beaucoup trop loin dans l'anticipation.

En réalité, à l'heure actuelle, seul l'emploi des rayons ultra-vio- 
lets approche de la phase d'application industrielle, pour ce qui regarde la stérilisation. Pour la mise en œuvre des autres radiations, de patientes recherches seront nécessaires, en vue de déterminer avec précision les possibilités d'emploi, et les résultats qu'il est légitime d'attendre de cette curieuse extension des méthodes de conservation des matières alimentaires.

\section{BIBLIOGRAPHIE}

[1] Voprossy Pitania, 1935, 4, 147.

[2] Journal Bact., 1942, 43, 125.

[3] Deutsche Molk, Ztg., 1941.

[4] Journal of Dairy Science, 1941, 24, 1055.

[5] Cold-ray pasteurisation of Milk. (Rapp. no 107 du British Intelligence Objectives Sub-Committee).

[6] Food Ind., 1947, 19, 1495.

[7] Milk Dealer, 1932, 21, 40.

[8] Journal Bact., 1946, 51, $\$ 87$.

[9] Deutsche Molk. Ztg., 1941, 45, 1052.

\section{ÉTUDE BACTÉRIOPHAGIQUE DU COLOSTRUM} par

\section{InèNe LIPSKA}

Au IVe Congrès de Microbiologie à Copenhague en 1947, j'ai eu la possibilité de rapporter les effets euratifs des bactériophages et j'ai indiqué les premiers essais préventifs contre les diarrhées d'été des nouveau-nés. Des bactériophages polyvalents furent administrés dans une première étude, le $4^{\mathrm{e}}$ et le $5^{\mathrm{e}}$ jour, et, dans une deuxième, le $2^{\mathrm{e}}$ et le $3^{\mathrm{e}}$ jour après la naissance. Une centaine de nouveau-nés, dans une clinique gynécologique ont reçu $2 \mathrm{~cm}^{3}$ des bactériophages polyvalents le $2^{\mathrm{e}}$ et le $3^{\mathrm{e}}$ jour. Après une année, ces nourrissons ont été visités par une même infirmière expérimentée pour constater leur état de santé. Les données furent que $71 \%$ n'avaient pas, pendant toute cette année, présenté de trouble digestif; $15 \%$ présentaient parfois des fèces moins moulés et $14 \%$ ont quitté Varsovie sans laisser d'adresse. Encouragée par ce bon résultat, j'ai recommencé à donner les bactériophages polyvalents, mais, cette fois, comme le premier liquide donné au nouveau-né. Dans une première période, j'ai ainsi traité soixante nouveau-nés ; encouragée, je crois devoir continuer ce travail.

Pour constater que le colostrum et le lait de la mère ne sont pas une source de bactériophages pour les nouveau-nés et n'ayant nulle part trouvé des études sur ce sujet, j'entrepris moi-même ce travail. 